\title{
ARTICLE OPEN \\ Connecting $n$th order generalised quantum Rabi models: Emergence of nonlinear spin-boson coupling via spin rotations
}

\author{
Jorge Casanova ${ }^{1}$, Ricardo Puebla ${ }^{1,2}$, Hector Moya-Cessa $\mathbb{D}^{3,4}$ and Martin B. Plenio ${ }^{1}$
}

\begin{abstract}
We establish an approximate equivalence between a generalised quantum Rabi model and its $n$th order counterparts, where spinboson interactions are nonlinear as they comprise a simultaneous exchange of $n$ bosonic excitations. Although there exists no unitary transformation between these models, we demonstrate their equivalence to a good approximation in a wide range of parameters. This shows that nonlinear spin-boson couplings, i.e., nth order quantum Rabi models, are accessible to quantum systems with only linear coupling between boson and spin modes by simply adding spin rotations and after an appropriate transformation. Furthermore, our result prompts novel approximate analytical solutions to the dynamics of the quantum Rabi model in the ultrastrong coupling regime improving previous approaches.
\end{abstract}

npj Quantum Information (2018)4:47; doi:10.1038/s41534-018-0096-9

\section{INTRODUCTION}

The quantum Rabi model (QRM) lies not only at the heart of our understanding of light-matter interaction, ${ }^{1}$ but is also of importance in diverse fields of research. ${ }^{2}$ The Rabi model was primarily proposed to describe a nuclear spin interacting with classical radiation, ${ }^{3,4}$ whose quantised version only appeared two decades later. ${ }^{5}$ This contemplates a scenario which is of great generality as it encompasses two of the most basic, yet essential, ingredients in quantum physics, namely, a two-level system and a bosonic mode. Indeed, this model emerges in disparate settings, ranging from ion traps ${ }^{6,7}$ to circuit or cavity $\mathrm{QED}_{1}^{8,9}$ quantum optomechanical systems, ${ }^{10}$ colour-centres in membranes, ${ }^{11}$ and cold atoms. $^{12}$

Even though the QRM has been exhaustively investigated in the last decades, a number of recent findings has brought it again into the research spotlight. Among them we can mention its integrability, ${ }^{13}$ the existence of a distinctive behaviour in the deep strong coupling regime, ${ }^{14}$ or the emergence of a quantum phase transition. ${ }^{15-18}$ Closely related to the QRM, we find the $n$th order QRM (nQRM) which differs from the QRM in that the nQRM comprises $n$-boson exchange interaction terms because of the presence of a nonlinear spin-boson coupling. This generalisation of the QRM has recently attracted attention, mainly in its secondorder form (2QRM) as it shows striking phenomena such as spectral collapse, ${ }^{19-21}$ due to its relevance in preparing nonclassical states of light in quantum optics 22,23 and regarding its solvability. ${ }^{24-26}$ These studies have also been extended to a mixed QRM comprising both one-boson and two-boson interaction terms, which appears in the context of circuit QED. ${ }^{27-29}$ Furthermore, solutions to this mixed QRM have recently been found, ${ }^{30}$ and it has also been reported that this model displays quantum phase transitions. ${ }^{31}$ Due to these compelling physical properties, the coherent control of nth order quantum Rabi models could open new avenues to develop different fields as quantum computing or quantum simulations. In addition, because of their different spectra, it is worth noting that there is no unitary map between the QRM and the nQRM with $n>1$.

In this article, we demonstrate the existence of a connection, i.e., an approximate equivalence, among a family of Hamiltonians comprising nth order boson interaction terms, where the standard QRM or the 2QRM appear as special cases. As a proof of concept, we show how the dynamics of a $2 \mathrm{QRM}$ and a 3QRM can be captured without having access to the required nonlinear twophoton and three-photon interactions, and after an appropriate transformation of a linear QRM that includes spin driving terms, i.e., spin rotations. The latter is dubbed here as generalised QRM (gQRM). In this manner we can argue that, a quantum system that contains a linear spin-boson coupling but lacks of nonlinear interactions suffices for the simulation of models where nonlinear terms are crucial. Our method works as follows: The dynamics of a state $|\psi\rangle$ evolving under a nQRM (the targeted dynamics) can be successfully retrieved from a gQRM (the starting point of our method) by (i) evolving a transformed initial state $T|\psi\rangle$ under gQRM during a time $t$ and (ii) measuring customary spin and boson observables of the gQRM. We will demonstrate that the latter corresponds to expectation values of observables of the state $|\psi(t)\rangle$ evolved under the nonlinear nQRM (see Fig. 1 for a scheme of the method). Indeed, as creating $n$-boson interactions is considered challenging in many quantum platforms, our method opens new avenues for their inspection. It is worth stressing that this reported method fundamentally differs from previous works where resonant multi-boson effective Hamiltonians were obtained, either via amplitude modulation as used in circuit $\mathrm{QED}^{32-34}$ or via adiabatic passage. ${ }^{35,36}$ In these works, effective multi-boson exchange terms do not comprise nonlinear spinboson couplings and hold only in a very limited parameter regime and/or for particular states. Certainly, in this article we report an approximate equivalence among nQRMs which holds in a large

\footnotetext{
${ }^{1}$ Institut für Theoretische Physik and IQST, Universität Ulm, Albert-Einstein Allee 11, 89069 Ulm, Germany; ${ }^{2}$ Centre for Theoretical Atomic, Molecular, and Optical Physics, School of

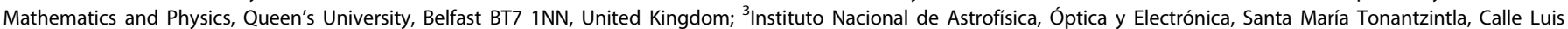
Enrique Erro 1, Puebla 72840, Mexico and ${ }^{4}$ Institut für Quantenphysik and IQST, Universität Ulm, Albert-Einstein-Allee 11, 89081 Ulm, Germany

Correspondence: Jorge Casanova (jcasanovamar@gmail.com) or Ricardo Puebla (rpueblaantunes@gmail.com)
}

Received: 31 May 2018 Revised: 31 August 2018 Accepted: 6 September 2018

Published online: 28 September 2018 


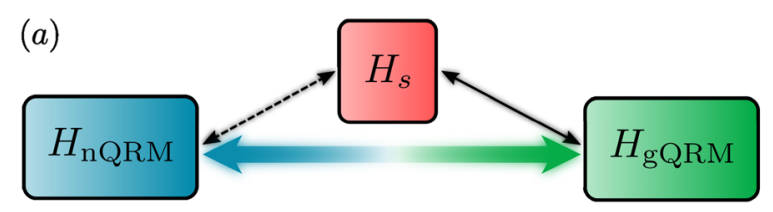

(b)

$$
\begin{aligned}
\left|\psi_{\mathrm{nQRM}}(0)\right\rangle & \\
\left|\psi_{\mathrm{nQRM}}(t)\right\rangle & \left|\psi_{\mathrm{gQRM}}(0)\right\rangle=T(i \eta / 2)\left|\psi_{\mathrm{nQRM}}(0)\right\rangle \\
O_{\mathrm{nQRM}} & \left|\psi_{\mathrm{gQRM}}(t)\right\rangle \approx \Gamma^{\dagger}(t)\left|\psi_{\mathrm{nQRM}}(t)\right\rangle \\
& O_{\mathrm{gQRM}}=\Gamma^{\dagger}(t) O_{\mathrm{nQRM}} \Gamma(t)
\end{aligned}
$$

Fig. 1 Scheme of the approximate equivalence. a Diagram of the approximate equivalence among $H_{s}, H_{\text {nQRM }}$ and $H_{\text {gQRM }}$. The transformation between $H_{\mathrm{s}}$ and $H_{\mathrm{gQRM}}$ is exact (solid arrow), while between $H_{\mathrm{s}}$ and $H_{\mathrm{nQRM}}$ is approximated (dashed arrow). Hence, we establish an approximate map between nQRM and gQRM (blurred arrow). $\mathbf{b}$ The latter is accomplished by the transformations between initial states $\left(\left|\psi_{\text {nQRM }}(0)\right\rangle,\left|\psi_{\text {gQRM }}(0)\right\rangle\right)$, evolved states $\left(\left|\psi_{\text {nQRM }}(t)\right\rangle, \mid\right.$ $\left.\left.\psi_{\mathrm{gQRM}}(t)\right\rangle\right)$, and observables ( $\left.O_{\mathrm{nQRM}}, O_{\mathrm{gQRM}}\right)$

range of parameters and grants a large tunability to explore their physics, as well as it unveils a fundamental relation between these models. Moreover, we present a potentially scalable platform, ${ }^{37}$ a microwave-driven trapped ion setting, ${ }^{38-41}$ in which nQRMs are unattainable without resorting to our approximate equivalence, which highlights the applicability of our method. Finally, we use our theory to analyse the standard QRM and find that our method provides, in addition, approximate analytical solutions that surpass in accuracy previous approaches in the ultrastrong coupling regime. $^{42-47}$

\section{RESULTS}

Description of the approximate equivalence

We begin with the following general Hamiltonian (later we will demonstrate its connection with the gQRM that only contains linear spin-boson interactions and represents the starting point of our approximate equivalence)

$H_{\mathrm{s}}=\nu a^{\dagger} a+\frac{\omega}{2} \sigma_{z}+\frac{\Omega}{2} \sum_{j}\left[\sigma^{+} e^{i \eta\left(a+a^{\dagger}\right)} e^{-i a_{j}}+\right.$ H.c $]$,

whose first two terms correspond to a bosonic mode of frequency $v$ and a two-level system with a frequency splitting $\omega$, described by the usual annihilation (creation) operator $a\left(a^{\dagger}\right)$ and spin- $\frac{1}{2}$ Pauli matrices $\vec{\sigma}=\left(\sigma_{x}, \sigma_{y}, \sigma_{z}\right)$. Both subsystems interact through a set of coupling terms with amplitude $\Omega / 2$ and parameter $\eta$, considered here equal $\forall j$, and $a_{j}$ being a time dependent phase. The Hamiltonian $H_{s}$ is central for our theory, as sketched in Fig. 1, and establishes an approximate map between gQRM dynamics with those of the nQRM. We perform a unitary transformation on $H_{\mathrm{s}}$ to find $H_{T}=T($ in $/ 2) H_{\mathrm{s}} T^{\dagger}($ in $/ 2)$, where $T(\beta)=$ $1 / \sqrt{2}\left[\mathcal{D}(\beta)(|e\rangle\langle g|+| g\rangle\langle g|)+\mathcal{D}^{\dagger}(\beta)(|e\rangle\langle e|-| g\rangle\langle e|)\right]$ with $\sigma_{z}=$ $|e\rangle\langle e|-| g\rangle\langle g|$ and $\mathcal{D}(\beta)=e^{\beta a^{\dagger}-\beta^{*} a}$ is the displacement operator. Note that this transformation has been used in the context of trapped ions to derive the eigenstates of a system that comprises a laser interacting with a trapped ion, and for fast implementations of the QRM. ${ }^{48,49}$ Now, choosing time dependent phases, $a_{j}=$ $\left(\omega+\delta_{j}\right) t$, and moving to a rotating frame with respect to $H_{T, 0}=$ $-\left(\omega+\delta_{1}\right) \sigma_{x} / 2$, the resulting Hamiltonian, $H_{\mathrm{gQRM}}$, reads (for more details see Methods section)

$$
\begin{aligned}
H_{\mathrm{gQRM}} \equiv & \mathcal{U}_{T, 0}^{\dagger}(t)\left(H_{T}-H_{T, 0}\right) \mathcal{U}_{T, 0}(t) \\
= & \nu a^{\dagger} a+\frac{\delta_{1}}{2} \sigma_{x}-\frac{\eta \nu}{2} p \sigma_{x} \\
& \quad+\frac{\Omega}{2} \sum_{j}\left\{\cos \left[\left(\delta_{j}-\delta_{1}\right) t\right] \sigma_{z}+\sin \left[\left(\delta_{j}-\delta_{1}\right) \mathrm{t}\right] \sigma_{\mathrm{y}}\right\}
\end{aligned}
$$

with $p=i\left(a^{\dagger}-a\right)$ and $\mathcal{U}_{T, 0}=e^{-i t H_{T, 0}}$. The previous Hamiltonian is the one of the gQRM, where its last term can be viewed as a classical driving acting on the system, i.e. this is the term leading to spin rotations. In particular, we note that $H_{\mathrm{gQRM}}$ adopts the form of a standard QRM in the case of having $\delta_{j}=0 \forall j$.

On the other hand, the Hamiltonian $H_{s}$ in Eq. (1) can be brought into the form of a $H_{\text {nQRM }}$ by properly choosing $a_{j}$ and in a suitable interaction picture. More specifically, by defining $H_{s}=H_{s, 0}+H_{s, 1}$ with $H_{s, 0}=(\nu-\tilde{\nu}) a^{\dagger} a+(\omega-\tilde{\omega}) \sigma_{z} / 2$ and considering two interaction terms (i.e., $j=1,2$ ) such that $\delta_{1,2}=\mp n \nu-\tilde{\omega} \pm n \tilde{\nu}$ (recall that $a_{j}=\left(\omega+\delta_{j}\right) t$ and thus $a_{j}$ and $\delta_{j}$ are related) with $\tilde{\omega}>0$ and $\tilde{\nu}>0$, we find that $H_{s, 1}^{\prime}=e^{i t H_{s, 0}} H_{s, 1} e^{-i t H_{s, 0}}$ approximately leads to

$$
H_{\mathrm{nQRM}}=\tilde{\nu} a^{\dagger} a+\frac{\tilde{\omega}}{2} \sigma_{z}+g_{n}\left[e^{i \phi_{n}} \sigma^{+}\left(a^{n}+\left(a^{\dagger}\right)^{n}\right)+\text { H.c }\right]
$$

with $\phi_{n}=n \pi / 2$ and $g_{n}=\eta^{n} \Omega /(2 n !)$. The validity of Eq. (3) is ensured when $\Omega \ll \nu, \quad|\tilde{\omega}+n \tilde{\nu}| \ll n \nu$ together with $|n| \sqrt{\left\langle\left(a+a^{\dagger}\right)^{2}\right\rangle} \ll 1$ to safely perform a rotating wave approximation (RWA) in the joint Hilbert space involving spin and bosonic degrees of freedom. In this respect, an expression of the leading order error committed by our scheme can be found in section I of Supplementary Information for further explanation and details of the calculation. The simulated nQRM can be brought into strong or ultrastrong coupling regimes as the parameters $\tilde{\omega}$ and $\tilde{\nu}$ can be tuned to frequencies comparable to $g_{n}$.

In this manner, having access to $H_{\mathrm{gQRM}}$ that includes only a linear spin-boson interaction, enables the exploration of a nQRM with nonlinear spin-boson coupling $(n>1)$, whose physics is fundamentally different. For example, the most exotic hallmarks of the two-photon QRM (2QRM), are that the spectrum becomes a continuum for $g_{2}=\tilde{\nu} / 2$ regardless of $\tilde{\omega}$, and for $g_{2}>\tilde{\nu} / 2$ the Hamiltonian is not longer lower bounded. ${ }^{19,20,25,50}$ The gQRM lacks these features, and it is therefore not obvious that the physics of $H_{2 Q R M}$ can be accessed from $H_{\mathrm{gQRM}}$. Moreover, the $H_{\mathrm{gQRM}}$ allows to simulate more exotic scenarios like combined nQRM and mQRM (see section II in Supplementary Information for further explanation and details of the calculation).

Based on the previous transformations one can find the following expression among operators that establishes a relation between the gQRM and nQRM dynamics, which is the central result of this article (see Methods for a more detailed derivation):

$\mathcal{U}_{\mathrm{gQRM}} \approx \Gamma^{\dagger}(t) \mathcal{U}_{\mathrm{nQRM}} T^{\dagger}($ in/2).

Here, $\mathcal{U}_{\text {gQRM }}$ and $\mathcal{U}_{\text {nQRM }}$ are the propagators of the gQRM and nQRM respectively, $\Gamma(t)=\mathcal{U}_{s, 0}^{\dagger} T^{\dagger}(i n / 2) \mathcal{U}_{T, 0}$ with $\mathcal{U}_{s, 0}=e^{-i t H_{s, 0}}$, and the approximate character of Eq. (4) is only a consequence of the RWA performed to achieve $H_{\text {nQRM }}$ from $H_{\mathrm{s}}$. Hence, an initial state | $\left.\psi_{\text {nQRM }}(0)\right\rangle$ after an evolution time $t$ under $H_{\text {nQRM }}$ can be approximated as $\left|\psi_{\mathrm{nQRM}}(t)\right\rangle \approx \Gamma(t)\left|\psi_{\mathrm{gQRM}}(t)\right\rangle$ with the initial state $\left.\psi_{\mathrm{gQRM}}(0)\right\rangle=T(\mathrm{in} / 2)\left|\psi_{\mathrm{nQRM}}(0)\right\rangle$.

Remarkably, while the dynamics under the gQRM occurs in a typical time $1 /(\eta v)$, see Eq. (2), the simulated nQRM (Eq. (3)) involves parameters that are much smaller than $v$ since they satisfy the previously commented conditions $\Omega \ll \nu$, $|\tilde{\omega}+n \tilde{\nu}| \ll n \nu$, and $g_{n}=\eta^{n} \Omega /(2 n$ !). As a consequence, a long evolution time of gQRM is required to effectively reconstruct the dynamics of nQRM.

Finally, our theory is completed with a mapping for the observables. As it can be derived from Eq. (4) (see Methods), the expectation value of an observable $O_{\text {nQRM }}$, i.e., an observable of the nQRM, corresponds to evaluate $O_{\mathrm{gQRM}}=\Gamma^{\dagger}(t) O_{\mathrm{nQRM}} \Gamma(t)$ in the gQRM. Because $\Gamma(t)$ involves bosonic displacement and spin rotations, $O_{\mathrm{gQRM}}$ may be in general intricate. Yet, for two relevant observables in nQRM, $\sigma_{z}$ and $a^{\dagger} a$, the mapping leads to simple operators, namely, $\sigma_{z}$ transforms into $-\sigma_{x}$ and $a^{\dagger} a$ into 
$a^{\dagger} a-\eta / 2 p \sigma_{x}+\eta^{2} / 4$ (see Methods). Interestingly, it still possible to obtain good approximations for other observables by truncating bosonic operators. Indeed, $e^{-\eta^{2} / 2}\left[\sigma_{z, y} \cos \left(\left(\tilde{\omega}+\delta_{1}\right) t\right) \mp\right.$ $\left.\sigma_{y, z} \sin \left(\left(\tilde{\omega}+\delta_{1}\right) t\right)\right]$ turns to be a good approximation of $\sigma_{x, y}$ in the gQRM frame (see Sec. III in Supplementary Information for further explanation and details of the calculation) which allows to recover the full qubit dynamics of $n Q R M$.

Approximate equivalence among gQRM and 2 and 3QRM

To numerically confirm our approximate equivalence, in Fig. 2 we show the results of the simulated dynamics of a $2 \mathrm{QRM}$ and a $3 Q R M$ using a gQRM for a certain set of parameters and initial states $\mid \psi$ $\left.(0)_{2 Q R M}\right\rangle=|2\rangle|\uparrow\rangle_{x} \quad$ and $\quad\left|\psi(0)_{3 Q R M}\right\rangle=(|0\rangle+|1\rangle) / \sqrt{2}|\uparrow\rangle_{x}$, with $|\uparrow(\downarrow)\rangle_{x}=(|e\rangle \pm|g\rangle) / \sqrt{2}$. In addition, in Fig. 2c we show that the targeted $\sigma_{x}$ of a nQRM is retrieved by means of the previously mentioned bosonic truncation of $\sigma_{x}$ in the gQRM frame, i.e., $e^{-\eta^{2} / 2}\left[\sigma_{z} \cos \left(\left(\tilde{\omega}+\delta_{1}\right) t\right)-\sigma_{y} \sin \left(\left(\tilde{\omega}+\delta_{1}\right) t\right)\right]$. Furthermore, in order to quantify the agreement among these models and the validity of the previous theory, we compute the fidelity between the ideal quantum state of the nQRM and the approximated state evolved in the gQRM and properly transformed with $\Gamma(t)$, that is, $F_{\mathrm{g}, n}(t)=\left\langle\psi_{\mathrm{gQRM}}(t)\left|\Gamma^{\dagger}(t)\right| \psi_{\mathrm{nQRM}}(t)\right\rangle$. The computed fidelities of the considered cases are well above 0.99, showing the good agreement among these two models. Note that although $\mathrm{H}_{3 \mathrm{QRM}}$ could present truncation problems for $g_{3} \neq 0$ (see ref. ${ }^{51}$ ), these do not affect the dynamics for the particular case plotted in Fig. 2. Indeed, for the chosen parameters and initial state, the dynamics during the considered evolution takes place in a constrained region of the Hilbert space and thus it does not show Fock space truncation problems (see section IV in Supplementary Information for further explanation and details of the calculation] for further details). It is however worth stressing that this is not the general case, because the $3 \mathrm{QRM}$ is not bounded from below. Therefore, the number of excitations can grow very fast and, as a consequence, the simulation of the $3 Q R M$ relying on the approximate equivalence will break down since $|\eta| \sqrt{\left\langle\left(a+a^{\dagger}\right)^{2}\right\rangle} \ll 1$ is not longer satisfied. It is important to mention that our approximate equivalence is, in addition, not restricted to small times. The latter assertion is corroborated in Fig. 2 where the propagators for the 2QRM and 3QRM for the final time $t_{f}=2 \pi \frac{4}{\tilde{\nu}}$ (values of $\tilde{\nu}$ in the caption) are $\exp \left\{-i \pi\left[\frac{\tilde{\nu}}{g_{2}} a^{\dagger} a+\frac{\tilde{\omega}}{2 g_{2}} \sigma_{z}-\sigma_{x}\left(a^{2}+\left(a^{\dagger}\right)^{2}\right)\right]\right\}$ and $\exp \left\{-i 2 \pi / 5\left[\frac{\tilde{\nu}}{g_{3}} a^{\dagger} a+\frac{\tilde{\omega}}{2 g_{3}} \sigma_{z}+\sigma_{y}\left(a^{3}+\left(a^{\dagger}\right)^{3}\right)\right]\right\}$ respectively. Note that in both previous cases the coupling terms are multiplied by a phase $\pi$ and $2 \pi / 5$ respectively. We furthermore stress that these phases $(\pi$ and $2 \pi / 5$ ) can be increased without deteriorating the achieved fidelities by simply choosing a larger value for $v$. As previously commented, this is indeed possible since the approximate character of our method appears when we equal $H_{s}$ to $H_{\text {nQRM, }}$, whose performance is enhanced for large values of $v$ (see Methods).

\section{Application for microwave driven ions}

The proof-of-concept of our method can be illustrated in a microwave driven ions platform. Note that the developed theory
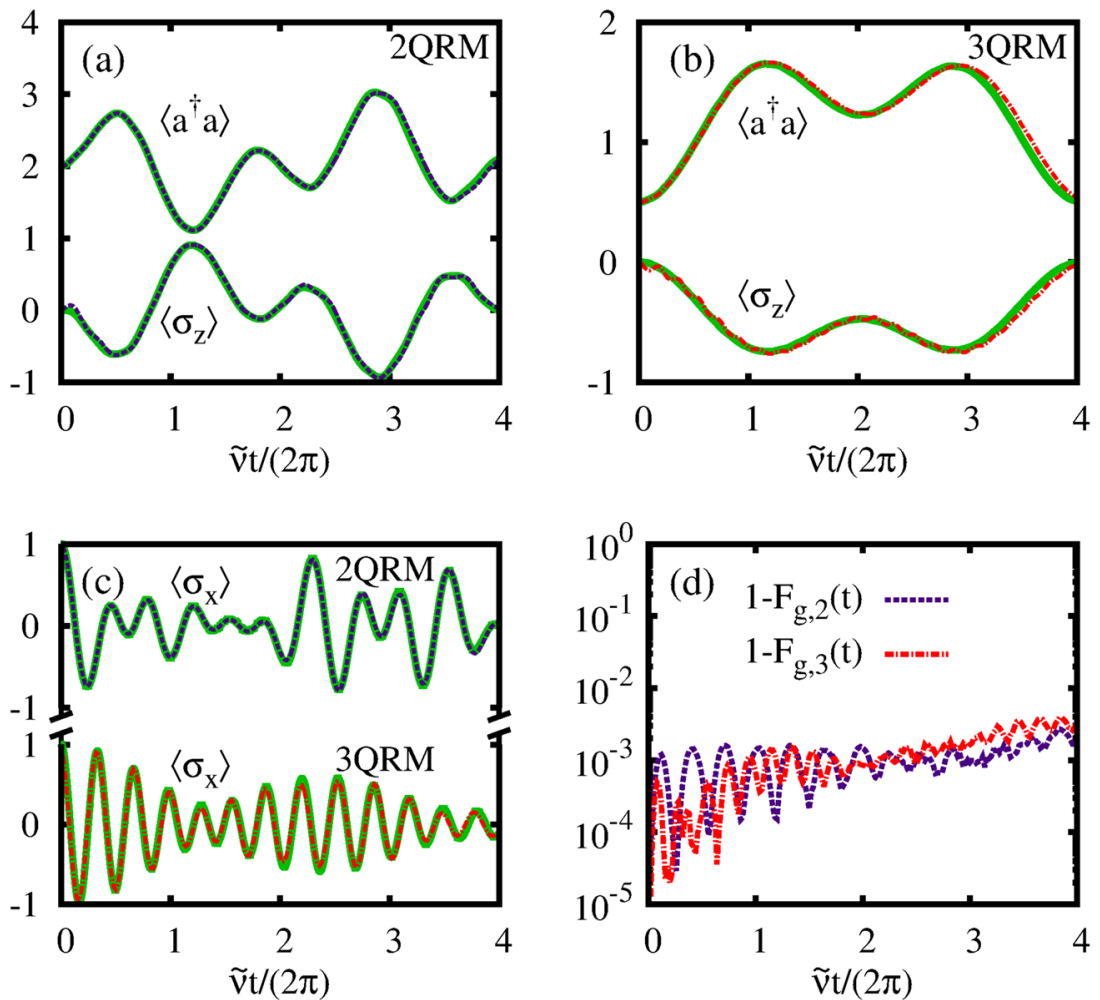

Fig. 2 Simulated $n Q R M$ dynamics using $g Q R M$. Comparison between the dynamics of $H_{\text {nQRM }}$ and the simulated one using $H_{\mathrm{gQRM}}$, for $n=2$ (a) and $n=3$ (b). a, b Show $\left\langle\sigma_{z}\right\rangle$ and $\left\langle a^{\dagger} a\right\rangle$ of the nQRM (solid green lines) and their counterpart in the gQRM frame, that is, $-\left\langle\sigma_{x}\right\rangle$ and $\left\langle a^{\dagger} a\right\rangle-\eta / 2\left\langle p \sigma_{x}\right\rangle+\eta^{2} / 4$, respectively, depicted with dashed dark blue (2QRM) and dotted-dashed red lines (3QRM). With the same style, in (c) we show the ideal $\left\langle\sigma_{x}\right\rangle$ for 2QRM (top) and 3QRM (bottom) and its approximated counterpart using gQRM, obtained through bosonic truncation (see main text). We take as initial state $\left|\psi_{2 \mathrm{QRM}}(0)\right\rangle=|2\rangle|\uparrow\rangle_{x}$ and the parameters $g_{2} / \tilde{\nu}=0.125$ and $\tilde{\omega}=2 \tilde{\nu}$. For the $3 \mathrm{QRM}$, $\left|\psi_{3 \mathrm{QRM}}(0)\right\rangle=(|0\rangle+|1\rangle) / \sqrt{2}|\uparrow\rangle_{x^{\prime}} g_{3} / \tilde{\nu}=0.05$ and $\tilde{\omega}=3 \tilde{\nu}$. For $H_{\mathrm{gQRM}}, \omega / \nu=10^{8}, \Omega / \nu=0.1$ and $\tilde{\nu} / \nu=5 \times 10^{-4}$. In d we show the infidelity between the states for the two considered models, $1-F_{\mathrm{g}, 2}(t)$ (dashed dark blue line) and $1-F_{\mathrm{g}, 3}(t)$ (dotted-dashed red line) 
may be relevant in other systems as circuit QED. ${ }^{9}$ A microwavedriven trapped ion in a magnetic field gradient is described by (for more details see ref. ${ }^{38-41}$ )

$H_{\mathrm{MW}}=\frac{\omega}{2} \sigma_{z}+\nu a^{\dagger} a+\Delta\left(a+a^{\dagger}\right) \sigma_{z}+\sum_{j} \Omega_{j} \sigma_{x} \cos \left(\omega_{j} t+\varphi_{j}\right)$,

where $\omega$ is the qubit energy splitting with a value that depends on the ion species. For example, for ${ }^{171} \mathrm{Yb}^{+}$, we have $\omega \approx 12.4 \mathrm{GHz}^{52}$ plus a factor $\gamma B_{z}$ with $\gamma \approx 1.4 \mathrm{MHz} / \mathrm{G}$ that depends on the applied static magnetic field $B_{z}$. The coupling parameter $\Delta$ determines the rate of the spin-boson coupling, while the last term corresponds to the action of microwave radiation on the system. ${ }^{53}$ In this setup the spin-boson coupling is restricted to be linear, and therefore our theory appears as an alternative to introduce higher-order boson couplings in the dynamics. In order to take Eq. (5) into the form of Eq. (2), and subsequently (via the mapping $T$ ) into the general expression in Eq. (1), we define $\omega=\delta_{1}+\tilde{\omega}$ and move to a rotating frame with respect to the term $\frac{\tilde{\omega}}{2} \sigma_{z}$. Considering two drivings such that $\varphi_{1,2}=\pi, \omega_{1}=\tilde{\omega}$ and $\omega_{2}=\tilde{\omega}-\left(\delta_{2}-\delta_{1}\right)$ and after eliminating terms that rotate at frequencies on the order of $\mathrm{GHz}$, we find

$H_{\mathrm{MW}}^{\prime}=\nu a^{\dagger} a+\Delta\left(a+a^{\dagger}\right) \sigma_{z}+\frac{\delta_{1}}{2} \sigma_{z}-\frac{\Omega}{2} \sigma_{x}-\frac{\Omega}{2}\left(\sigma^{+} e^{i\left(\delta_{2}-\delta_{1}\right) t}+\right.$ H.c. $)$,

which equals $H_{\mathrm{gQRM}}$ after a basis change, that is, $e^{-i \frac{\pi}{4} \sigma_{y}} e^{-i \frac{\pi}{2} a^{\dagger} a} H_{\mathrm{MW}}^{\prime} e^{i \frac{\pi}{2} a^{\dagger} a} e^{i \frac{\pi}{4} \sigma_{y}}=H_{\mathrm{gQRM}}$, where $H_{\mathrm{gQRM}}$ is given in Eq. (2) with $\eta=2 \Delta / v$. Hence, it is possible to use a microwave-driven ion to simulate models with nonlinear spin-boson couplings (see section V in Supplementary Information for further explanation and details of the calculation] for more details concerning the implementation in this setup).

Approximate analytical solution for the QRM

Finding a solution to the QRM has been subject of a long-standing debate, which still attracts considerable attention..$^{13,25,54,55}$ Based on our theory, we obtain a simple expression for the timeevolution propagator and expectation values of the QRM. The general expression given in Eq. (2) adopts the form of a standard QRM with a unique driving and $\delta_{1}=0$,

$H_{\mathrm{QRM}}=H_{\mathrm{gQRM}}\left(\delta_{1}=0\right)=\nu a^{\dagger} a-\frac{\eta \nu}{2} p \sigma_{x}+\frac{\Omega}{2} \sigma_{z}$,

which, applying our method, approximately corresponds to $H_{\text {aux }}=\Omega / 2 \sigma_{x}\left[1-\eta^{2}\left(a^{\dagger} a+1 / 2\right)\right]$. Indeed, from $H_{s}=H_{s, 0}+H_{s, 1}$ with $H_{s, 0}=\nu a^{\dagger} a+\omega \sigma_{z} / 2$ (setting $\tilde{\omega}=\tilde{\nu}=0$ ), we obtain now $\mathcal{U}_{s, 0}^{\dagger} H_{s, 1} \mathcal{U}_{s, 0} \approx H_{\text {aux }}$ instead of $H_{\text {nQRM }}$, and where fast oscillating terms have been neglected performing a RWA, requiring again $|\eta| \sqrt{\left\langle\left(a+a^{\dagger}\right)^{2}\right\rangle} \ll 1$, and only considering resonant terms up to $\eta^{2}$ (see Sec. IV in Supplementary Information for further explanation and details of the calculation). As a consequence, the following analysis does not apply to the deep-strong coupling regime, ${ }^{14}$ found here when $\eta \geq 2$. Hence, the propagator for the QRM is approximated as

$\mathcal{U}_{\mathrm{QRM}} \approx \mathcal{U}_{T, 0}^{\dagger} T\left(\right.$ in/2) $\mathcal{U}_{\mathrm{s}, 0} \mathcal{U}_{\text {aux }} T^{\dagger}($ in/2),

which is expected to hold even in the ultrastrong coupling regime of the QRM, although restricted to the condition $\Omega \ll \nu$. Because $H_{\text {aux }}$ has a simple form, the time evolution can be analytically solved, with an initial state $\left|\psi_{\text {aux }}(0)\right\rangle=T^{\dagger}($ in $/ 2)\left|\psi_{\text {ORM }}(0)\right\rangle$. Indeed, $H_{\text {aux }}=\sum_{n, \pm} E_{n}^{ \pm}\left|\varphi_{n}^{ \pm}\right\rangle\left\langle\varphi_{n}^{ \pm}\right| \quad$ with $\quad\left|\varphi_{n}^{ \pm}\right\rangle=|n\rangle|\uparrow(\downarrow)\rangle_{x} \quad$ and $E_{n}^{ \pm}= \pm \Omega / 2\left(1-\eta^{2}(n+1 / 2)\right)$. Now, employing the map between the two models, Eq. (8), we obtain the relation between observables. For example, $a^{\dagger} a$ in the QRM translates to $a^{\dagger} a+\eta^{2} / 4+$ $\eta / 2\left(x \sigma_{z} \sin \nu t-p \sigma_{z} \cos \nu t\right)$ in $H_{\text {aux }}$ (see section VII in Supplementary Information for further explanation and details of the calculation). In addition, we show that our method improves the typical Bloch-Siegert (BS) approximation ${ }^{43,44}$ and the generalised RWA (GRWA) of the $\mathrm{QRM}^{45-47}$ in a particular parameter regime. The former, i.e., the BS, is found as $e^{-S} H_{\mathrm{QRM}} e^{S} \approx H_{\mathrm{BS}}$, with $H_{\mathrm{BS}}=\left(\nu+\tilde{g} \wedge \sigma_{z}\right) a^{\dagger} a+(\Omega+\tilde{g} \Lambda) / 2 \sigma_{z}-\tilde{g}\left(i a^{\dagger} \sigma^{-}-i a \sigma^{+}\right)$, where the anti-Hermitian operator is given by $S=i \Lambda\left(a^{\dagger} \sigma_{+}+a \sigma_{-}\right)-\xi \sigma_{z}\left(a^{2}-\left(a^{\dagger}\right)^{2}\right), \quad$ with parameters $\Lambda=\tilde{g} /(\nu+\Omega), \xi=\tilde{g} \Lambda /(2 \nu)$ and $\tilde{g}=\eta \nu / 2$ (see ref. $\left.{ }^{43,44}\right)$. The GRWA of the QRM is attained in a similar manner, but with $S=$ $\tilde{g} / \nu X \sigma_{z}\left(a^{\dagger}-a\right)$ such that $e^{-S} H_{\mathrm{QRM}} e^{S} \approx H_{\mathrm{GRWA}}$, where $H_{\mathrm{GRWA}}$ has a Jaynes-Cummings form with modified parameters, see ref. ${ }^{45-47}$ and section VIII in the Supplementary Information for further explanation and details of the calculation] for furtherdetails. In Fig. 3 we compute the overlap between time-evolved states for these three approaches (our approximate solution, the BS approximation, and the GRWA) and the QRM. The approximate solution reproduces correctly the time evolution of the QRM as the coupling enters in the nonperturbative ultrastrong regime, $\tilde{g} / \nu=0.2$ (see ref. ${ }^{44}$ ) with a fidelity $F_{\mathrm{QRM} \text {,aux }}>0.99$, while approximations $H_{\mathrm{BS}}$ and $H_{\mathrm{GRWA}}$ fail as their fidelities drop significantly. For smaller couplings these approaches lead to similar high fidelities (see Fig. 3a).

\section{DISCUSSION}

We have presented a connection, i.e., an approximate equivalence, among a family of Hamiltonians, including the QRM and its higher order counterparts (nQRM) comprising a nonlinear interaction term that involves the simultaneous exchange of $n$ bosonic
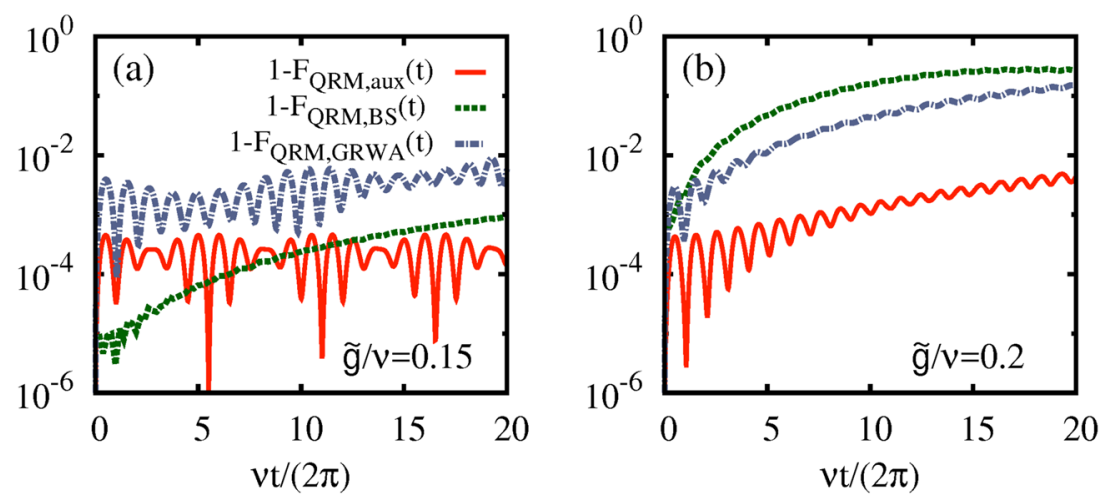

Fig. 3 Approximate solutions to the QRM. Infidelity between the time-evolved state of QRM and its approximate solution evolved from $H_{\text {aux }}$ the BS Hamiltonian $H_{\mathrm{BS}}$ and the GRWA approach $H_{\mathrm{GRWA}}$. These are denoted by $1-F_{\mathrm{QRM} \text {, aux }}$ (solid red), $1-F_{\mathrm{QRM}, B S}\left(\right.$ dashed green) and $1-F_{\mathrm{QRM}}$ GRWA (dashed-dotted blue), respectively. For $\mathbf{a} \Omega / \nu=0.1, \tilde{g} / \nu=\eta / 2=0.15$ and $|\psi(0)\rangle=|0\rangle|\uparrow\rangle_{x^{\prime}}$ and in $\mathbf{b} \Omega / \nu=0.04, \eta / 2=0.2$ and $|\psi(0)\rangle=|2\rangle \mid$ g) 
excitations with the spin-qubit, such as the two-photon QRM. In particular, the standard QRM including spin driving terms, i.e., the gQRM, allows us to retrieve the nQRM dynamics with very high fidelities. This theoretical framework shows that nQRMs can be accessed even in the absence of the required nonlinear spinboson exchange terms, as illustrated with a microwave-driven trapped ion. Therefore, we find that this fundamental model, the $\mathrm{gQRM}$, approximately contains the dynamics of all other nth order models. Moreover, we have derived an approximate solution to the dynamics of the QRM even in the ultrastrong coupling regime which surpasses in accuracy previous approximate solutions. In this manner, we have defined a general theoretical frame for the study and understanding of this family of fundamental Hamiltonians and their associated dynamics, which may open new avenues in quantum computing and simulation.

\section{METHODS}

Transformation between $H_{s}, H_{\mathrm{gQRM}}$, and $H_{\mathrm{nQRM}}$

The Hamiltonian $H_{s}$ given in Eq. (1), after the unitary transformation $H_{T}=T(i \eta / 2) H_{s} T^{\dagger}(i n / 2)$, adopts the following form

$H_{T}=\nu a^{\dagger} a-\frac{\omega}{2} \sigma_{x}+\frac{i \eta \nu}{2}\left(a-a^{\dagger}\right) \sigma_{x}+\frac{\nu \eta^{2}}{4}+\frac{\Omega}{2} \sum_{j}\left[\cos a_{j} \sigma_{z}+\sin a_{j} \sigma_{y}\right]$,

which becomes $H_{\text {gQRM }}$ in the rotating frame with respect to $H_{T, 0}=-(\omega+$ $\left.\delta_{1}\right) \sigma_{X} / 2$, namely, $H_{\mathrm{gQRM}} \equiv \mathcal{U}_{T, 0}^{\dagger}(t)\left(H_{T}-H_{T, 0}\right) \mathcal{U}_{T, 0}(t)$ as given in Eq. (2). For simplicity, we constrain ourselves to the case in which $\Omega_{j} \equiv \Omega \forall j$, although the procedure can be easily extended to a more general scenario. On the other hand, $H_{s}$ leads to the desired nQRM when moving to an interaction picture with respect to $H_{s, 0}=(\nu-\tilde{\nu}) a^{\dagger} a+(\omega-\tilde{\omega}) / 2 \sigma_{z}$ with $H_{s}=H_{s, 0}+$ $H_{s, 1}$ Then, the interacting part of $H_{s}$ can be written as

$$
\begin{aligned}
H_{s, 1}^{\prime} & \equiv \mathcal{U}_{s, 0}^{\dagger}\left(t, t_{0}\right)\left(H_{s}-H_{s, 0}\right) \mathcal{U}_{s, 0}^{\dagger}\left(t, t_{0}\right) \\
& =\tilde{\nu} a^{\dagger} a+\frac{\tilde{\omega}}{2} \sigma_{z}+\sum_{j} \frac{\Omega}{2}\left\{\sigma^{+} e^{i(\omega-\tilde{\omega}) t \prime} e^{\left.i \eta(a(t))+a^{\dagger}(t)\right)} e^{-i a_{j}}+\text { H.c. }\right\},
\end{aligned}
$$

with $a(t)=a e^{-i(\nu-\tilde{\nu}) t}, a^{\dagger}(t)=a^{\dagger} e^{i(\nu-\tilde{\nu}) t}$, and $\mathcal{U}_{s, 0}\left(t, t_{0}\right)$ the time-evolution operator associated to $H_{s, 0}$ such that $t^{\prime}=t-t_{0}$. Then, expanding the exponential, considering that $\Omega \ll \nu$ and $|\eta| \sqrt{\left\langle\left(a+a^{\dagger}\right)^{2}\right\rangle} \ll 1$, and that $\delta_{1,2}=\mp n \nu-\tilde{\omega} \pm n \tilde{\nu}$ with $|\tilde{\omega}+n \tilde{\nu}| \ll n \nu$, one can perform a rotating wave approximation just keeping those terms resonant with $\sigma^{+} a^{n}$ and $\sigma^{-} a^{n}$. In general,

$H_{s, 1}^{\prime} \approx H_{\mathrm{nQRM}}=\tilde{\nu} a^{\dagger} a+\frac{\tilde{\omega}}{2} \sigma_{z}+g_{n}\left[e^{i \phi_{n}} \sigma^{+}+e^{-i \phi_{n}} \sigma^{-}\right] \times\left[a^{n}+\left(a^{\dagger}\right)^{n}\right]$,

with $g_{n}=\eta^{n} \Omega /(2 n !)$ and $\phi_{n}=n \pi / 2$. Hence, it is possible to achieve a $H_{\text {nQRM }}$ from $H_{s}$. Note however that the corresponding attained coupling $g_{n}$ becomes smaller for increasing $n$, as it is proportional to $\eta^{n} / n$ !. In particular, for $n=2, H_{s, 1}^{\prime}$ can be approximated as

$H_{s, 1}^{\prime} \approx H_{2 \mathrm{QRM}}=\tilde{\nu} a^{\dagger} a+\frac{\tilde{\omega}}{2} \sigma_{z}-\frac{\eta^{2} \Omega}{4} \sigma_{x}\left(a^{2}+\left(a^{\dagger}\right)^{2}\right)$.

Note that, while the Hamiltonians $H_{s}$ and $H_{\mathrm{gQRM}}$ are related through a unitary transformation, the achievement of a n-photon QRM, $H_{\text {nORM }}$, from $H_{s}$ requires of certain relations between parameters, such as $\Omega \ll \nu, \tilde{\omega}+$ $n \tilde{\nu} \mid \ll n \nu$ and $|\eta| \sqrt{\left\langle\left(a+a^{\dagger}\right)^{2}\right\rangle} \ll 1$ to safely perform the rotating wave approximation. In addition, it is worth stressing that the equivalence to a good approximation is not restricted to $H_{\text {nQRM }}$ and $H_{\text {gQRM. }}$. For example, a $H_{\text {gQRM }}$ can lead into a more complex Hamiltonian, such as one comprising both $\mathrm{nQRM}$ and mQRM interaction terms (see Supplementary Information for further explanation and details of the calculation).

\section{Transformations of observables and states}

Here we show the derivation of the Eq. (4) which is a central result of this article. Having established the transformations that connect $H_{\mathrm{gQRM}}$ with $H_{s}$, and $H_{\text {nQRM }}$ with $H_{s}$ we can relate them in terms of the time-evolution operators,

$\mathcal{U}_{T}=T(i \eta / 2) \mathcal{U}_{s} T^{\dagger}(i \eta / 2)$

$\mathcal{U}_{T}=\mathcal{U}_{T, 0} \mathcal{U}_{T, 1}^{l}=\mathcal{U}_{T, 0} \mathcal{U}_{\mathrm{gQRM}}$

$\mathcal{U}_{s}=\mathcal{U}_{s, 0} \mathcal{U}_{s, 1}^{\prime} \approx \mathcal{U}_{s, 0} \mathcal{U}_{\text {nQRM }}$,

where $\mathcal{U}_{x, 1}^{\prime}$ denotes the time-evolution propagator of $H_{x, 1}$ in an interaction picture with respect to $H_{x, 0}$ such that $H_{x}=H_{s, 0}+H_{s, 1}$. Note that we have dropped the explicit time dependence for the sake of readability (see previous Eqs. (9-11) for the specific transformations). Then, combining the Eqs. (13-15), we arrive to

$\mathcal{U}_{\text {gQRM }} \approx \mathcal{U}_{T, 0}^{\dagger} T($ in $/ 2) \mathcal{U}_{s, 0} \mathcal{U}_{\text {nQRM }} T^{\dagger}($ in $/ 2)$,

which is the Eq. (4), $\mathcal{U}_{\mathrm{gQRM}}=\Gamma^{\dagger}(t) \mathcal{U}_{\mathrm{nQRM}} T^{\dagger}($ in/2) with $\Gamma(t)=\mathcal{U}_{s, 0}^{\dagger} T^{\dagger}(i \eta / 2) \mathcal{U}_{T, 0}$. Then,

$$
\begin{aligned}
\left|\psi_{\mathrm{nQRM}}(t)\right\rangle & =\mathcal{U}_{\mathrm{nQRM}}\left|\psi_{\mathrm{nQRM}}(0)\right\rangle \\
& \approx \Gamma(t) \mathcal{U}_{\mathrm{gQRM}} T(i \eta / 2)\left|\psi_{\mathrm{nQRM}}(0)\right\rangle \\
& =\Gamma(t)\left|\psi_{\mathrm{gQRM}}(t)\right\rangle
\end{aligned}
$$

with the relation between initial states $\left|\psi_{\mathrm{gQRM}}(0)\right\rangle=T(i \eta / 2)\left|\psi_{\mathrm{nQRM}}(0)\right\rangle$. Finally, from Eq. (17) it is straightforward to obtain the observable that must be measured in the gQRM frame in order to retrieve $O_{\text {nORM }}$ of the nQRM, i.e., $O_{g Q R M}=\Gamma^{\dagger}(t) O_{\text {nQRM }} \Gamma(t)$. Explicitly, $\Gamma(t)$ reads

$\Gamma(t)=e^{-i t(\tilde{\omega}-\omega) / 2 \sigma_{z}} e^{-i t(\tilde{\nu}-\nu) a^{\dagger} a} T^{\dagger}(i \eta / 2) e^{-i t\left(-\left(\omega+\delta_{1}\right) / 2 \sigma_{x}\right)}$

and thus, for $O_{\mathrm{nQRM}}=\sigma_{z}$ and $a^{\dagger} a$ the transformation leads to

$\left(\sigma_{z}\right)_{\mathrm{gQRM}}=-\sigma_{x}$

$\left(a^{\dagger} a\right)_{\mathrm{gQRM}}=a^{\dagger} a-\frac{\eta}{2} p \sigma_{x}+\frac{\eta^{2}}{4}$,

while for other observables, like $\sigma_{x}$ and $\sigma_{y}$, a more intricate expression is attained,

$$
\begin{aligned}
\left(\sigma_{x}\right)_{\mathrm{gQRM}}=\{ & \left.\cos \left(\left(\omega+\delta_{1}\right) t\right) \sigma_{z}-\sin \left(\left(\omega+\delta_{1}\right) t\right) \sigma_{y}\right\} \operatorname{Re}\left[\mathcal{D}(i \eta) e^{i(\omega-\tilde{\omega}) t}\right] \\
& +\left\{\sin \left(\left(\omega+\delta_{1}\right) t\right) \sigma_{z}+\cos \left(\left(\omega+\delta_{1}\right) t\right) \sigma_{y}\right\} \operatorname{Im}\left[\mathcal{D}(i \eta) e^{i(\omega-\tilde{\omega}) t}\right]
\end{aligned}
$$

$$
\begin{aligned}
\left(\sigma_{y}\right)_{\mathrm{gQRM}}=\{ & \left.\sin \left(\left(\omega+\delta_{1}\right) t\right) \sigma_{z}+\cos \left(\left(\omega+\delta_{1}\right) t\right) \sigma_{y}\right\} \operatorname{Re}\left[\mathcal{D}(i \eta) e^{i(\omega-\tilde{\omega}) t}\right] \\
& -\left\{\cos \left(\left(\omega+\delta_{1}\right) t\right) \sigma_{z}-\sin \left(\left(\omega+\delta_{1}\right) t\right) \sigma_{y}\right\} \operatorname{Im}\left[\mathcal{D}(i \eta) e^{i(\omega-\tilde{\omega}) t}\right]
\end{aligned}
$$

as it involves qubit and bosonic operators due to the presence of the displacement operator $\mathcal{D}(\beta)$. However, because the condition $|\eta| \sqrt{\left\langle\left(a+a^{\dagger}\right)^{2}\right\rangle} \ll 1$ is required to guarantee a good realisation of $H_{\text {nQRM }}$ and so that of Eq. (4), the previous expression can be well approximated by truncating $\mathcal{D}(\beta)$. Indeed, in our case $\mathcal{D}$ (in) can be approximated up to third order as

$$
\mathcal{D}(i \eta)=e^{-\eta^{2} / 2}\left[1+i \eta\left(a+a^{\dagger}\right)-\frac{\eta^{2}}{2}\left(2 a^{\dagger} a+\left(a^{\dagger}\right)^{2}+a^{2}\right)+\mathcal{O}\left(\eta^{3} a^{3}\right)\right]
$$

In general, we can approximate the observable $\left(\sigma_{j}\right)_{\mathrm{gQRM}}$ by truncating at order $M$, that is,

$$
\left(\sigma_{j}\right)_{\mathrm{gQRM}} \approx\left(\sigma_{j}\right)_{\mathrm{gQRM}}^{M}=\sum_{n=0}^{M}\left(\sigma_{j}\right)_{\mathrm{gQRM}}^{(n)},
$$

where the terms $\left(\sigma_{j}\right)_{\text {gORM }}^{(n)}$ for $j=x, y$ and can be calculated from Eqs. (2022). In particular, for $\sigma x, y$ and for $n=0$,

$$
\begin{aligned}
& \left(\sigma_{x}\right)_{\mathrm{gQRM}}^{(0)}=e^{-\eta^{2} / 2}\left[\sigma_{z} \cos \left(\left(\tilde{\omega}+\delta_{1}\right) t\right)-\sigma_{y} \sin \left(\left(\tilde{\omega}+\delta_{1}\right) t\right)\right] \\
& \left(\sigma_{y}\right)_{\mathrm{gQRM}}^{(0)}=e^{-\eta^{2} / 2}\left[\sigma_{z} \sin \left(\left(\tilde{\omega}+\delta_{1}\right) t\right)+\sigma_{y} \cos \left(\left(\tilde{\omega}+\delta_{1}\right) t\right)\right]
\end{aligned}
$$

Note that measuring $\left(\sigma_{x, y}\right)_{\mathrm{gORM}}^{(M)}$ would require measurements of observables in the gQRM of the form $\sigma_{y z}\left(a^{M}+\left(a^{\dagger}\right)^{M}\right)$ as well as $\sigma_{y, z}\left(a^{\dagger}\right)^{n} a^{m}$ with $n+m=M$ and $n \geq m$ (see Supplementary Information for further explanation and details of the calculation). Remarkably, for the considered cases here, the zeroth order approximation already reproduces reasonably well the expectation value of $\sigma_{x, y}$ of a nQRM. Therefore, having 
access to qubit observables in gQRM, $\sigma_{x, y, z}$ allows to reconstruct the full qubit dynamics of a nQRM. Note that Eqs. (24) and (25) correspond to the expressions given in Results, which for $\sigma_{x}$ is plotted in Fig. 2c for the simulation of a $2 \mathrm{QRM}$ and $3 \mathrm{QRM}$.

\section{DATA AVAILABILITY}

The data files used to prepare the figures shown in the manuscript are available from the first corresponding author upon request.

\section{ACKNOWLEDGEMENTS}

This work was supported by the ERC Synergy grant BioQ, the EU STREP project EQUAM. The authors acknowledge support by the state of Baden-Württemberg through bwHPC and the German Research Foundation (DFG) through grant no INST 40/467-1 FUGG. J. C. acknowledges Universität Ulm for a Forschungsbonus and support by the Juan de la Cierva grant IJCI-2016-29681. H. M.-C. thanks the Alexander von Humboldt Foundation for support. R. P. acknowledges DfE-SFI Investigator Programme (grant 15/IA/2864).

\section{AUTHOR CONTRIBUTIONS}

J. C. and R. P. have contributed equally to this work. J.C. and R.P. conceived the idea and develop the theory with inputs from H. M.-C. and M. B. P. All authors contributed to the writing of the manuscript.

\section{ADDITIONAL INFORMATION}

Supplementary information accompanies the paper on the npj Quantum Information website (https://doi.org/10.1038/s41534-018-0096-9).

Competing interests: The authors declare no competing interests.

Publisher's note: Springer Nature remains neutral with regard to jurisdictional claims in published maps and institutional affiliations.

\section{REFERENCES}

1. Scully, M. O. \& Zubairy, M. S. Quantum Optics. (Cambridge University Press, Cambridge, 1997).

2. Braak, D., Chen, Q.-H., Batchelor, M. T. \& Solano, E. Semi-classical and quantum Rabi models: in celebration of 80 years. J. Phys. A 49, 300301 (2016).

3. Rabi, I. I. On the process of space quantization. Phys. Rev. 49, 324-328 (1936).

4. Rabi, I. I. Space quantization in a gyrating magnetic field. Phys. Rev. 51, 652-654 (1937).

5. Jaynes, E. T. \& Cummings, F. W. Comparison of quantum and semiclassical radiation theories with application to the beam maser. Proc. IEEE 51, 89-109 (1963).

6. Leibfried, D., Blatt, R., Monroe, C. \& Wineland, D. Quantum dynamics of single trapped ions. Rev. Mod. Phys. 75, 281-324 (2003).

7. Häffner, H., Roos, C. F. \& Blatt, R. Quantum computing with trapped ions. Phys. Rep. 469, 155-203 (2008).

8. Haroche, S. \& Raimond, J.-M. Exploring the Quantum: Atoms, Cavities, and Photons. (Oxford University Press, Oxford, 2006).

9. Devoret, M. H. \& Schoelkopf, R. J. Superconducting circuits for quantum information: an outlook. Science 339, 1169-1174 (2013).

10. Aspelmeyer, M., Kippenberg, T. J. \& Marquardt, F. Cavity optomechanics. Rev. Mod. Phys. 86, 1391-1452 (2014).

11. Abdi, M., Hwang, M.-J., Aghtar, M. \& Plenio, M. B. Spin-mechanical scheme with color centers in hexagonal boron nitride membranes. Phys. Rev. Lett. 119, 233602 (2017).

12. Schneeweiss, P., Dareau, A. \& Sayrin, C. Cold-atom based implementation of the quantum Rabi model. https://arxiv.org/abs/1706.07781 (2017).

13. Braak, D. Integrability of the Rabi model. Phys. Rev. Lett. 107, 100401 (2011).

14. Casanova, J., Romero, G., Lizuain, l., Garca-Ripoll, J. J. \& Solano, E. Deep strong coupling regime of the Jaynes-Cummings model. Phys. Rev. Lett. 105, 263603 (2010).

15. Hwang, M.-J., Puebla, R. \& Plenio, M. B. Quantum phase transition and universal dynamics in the Rabi model. Phys. Rev. Lett. 115, 180404 (2015).

16. Puebla, R., Hwang, M.-J. \& Plenio, M. B. Excited-state quantum phase transition in the Rabi model. Phys. Rev. A 94, 023835 (2016).

17. Puebla, R., Hwang, M.-J., Casanova, J. \& Plenio, M. B. Probing the dynamics of a superradiant Quantum phase transition with a single trapped ion. Phys. Rev. Lett. 118, 073001 (2017)
18. Puebla, R., Casanova, J. \& Plenio, M. B. A robust scheme for the implementation of the quantum Rabi model in trapped ions. New J. Phys. 18, 113039 (2016).

19. Felicetti, S. et al. Spectral collapse via two-phonon interactions in trapped ions. Phys. Rev. A 92, 033817 (2015).

20. Duan, L., Xie, Y.-F., Braak, D. \& Chen, Q.-H. Two-photon Rabi model: analytic solutions and spectral collapse. J. Phys. A 49, 464002 (2016).

21. Puebla, R., Hwang, M.-J., Casanova, J. \& Plenio, M. B. Protected ultrastrong coupling regime of the two-photon quantum Rabi model with trapped ions. Phys. Rev. A 95, 063844 (2017).

22. Brune, M., Raimond, J. M., Goy, P., Davidovich, L. \& Haroche, S. Realization of a two-photon maser oscillator. Phys. Rev. Lett. 59, 1899-1902 (1987).

23. Toor, A. H. \& Zubairy, M. S. Validity of the effective Hamiltonian in the two-photon atom-field interaction. Phys. Rev. A 45, 4951-4959 (1992).

24. Travěnec, I. Solvability of the two-photon Rabi Hamiltonian. Phys. Rev. A 85, 043805 (2012).

25. Chen, Q.-H., Wang, C., He, S., Liu, T. \& Wang, K.-L. Exact solvability of the quantum Rabi model using Bogoliubov operators. Phys. Rev. A 86, 023822 (2012).

26. Cui, S., Cao, J.-P., Fan, H. \& Amico, L. Exact analysis of the spectral properties of the anisotropic two-bosons Rabi model. J. Phys. A 50, 204001 (2017).

27. Bertet, P. et al. Dephasing of a superconducting qubit induced by photon noise. Phys. Rev. Lett. 95, 257002 (2005).

28. Bertet, P., Chiorescu, I., Harmans, C. J. P. M. \& Mooij, J. E. Dephasing of a flux-qubit coupled to a harmonic oscillator. https://arxiv.org/abs/cond-mat/0507290.

29. Felicetti, S., Rossatto, D. Z., Rico, E., Solano, E. \& Forn-Díaz, P. Two-photon quantum Rabi model with superconducting circuits. Phys. Rev. A 97, 013851 (2018).

30. Duan, L., Xie, Y.-F. \& Chen, Q.-H. Solutions to the mixed quantum Rabi model. https://arxiv.org/abs/1807.02676.

31. Ying, Z.-J., Cong, L. \& Sun, X.-M. Quantum phase transition and spontaneous symmetry breaking in a nonlinear quantum Rabi model. https://arxiv.org/abs/ 1804.08128.

32. Strand, J. D. et al. First-order sideband transitions with flux-driven asymmetric transmon qubits. Phys. Rev. B 87, 220505 (2013).

33. Allman, M. S. et al. Tunable resonant and nonresonant interactions between a phase qubit and LC resonator. Phys. Rev. Lett. 112, 123601 (2014).

34. Lü, Z., Zhao, C. \& Zheng, H. Quantum dynamics of two-photon quantum Rabi model. J. Phys. A 50, 074002 (2017).

35. Ma, K. K. W. \& Law, C. K. Three-photon resonance and adiabatic passage in the large-detuning Rabi model. Phys. Rev. A 92, 023842 (2015).

36. Garziano, L. et al. Multiphoton quantum Rabi oscillations in ultrastrong cavity QED. Phys. Rev. A 92, 063830 (2015).

37. Lekitsch, B. et al. Blueprint for a microwave trapped ion quantum computer. Sci. Adv. http://advances.sciencemag.org/content/3/2/e1601540 (2017).

38. Mintert, F. \& Wunderlich, C. Ion-trap Quantum logic using long-wavelength radiation. Phys. Rev. Lett. 87, 257904 (2001).

39. Timoney, N. et al. Quantum gates and memory using microwave-dressed states. Nature 476, 185-188 (2011).

40. Weidt, S. et al. Trapped-ion Quantum logic with global radiation fields. Phys. Rev. Lett. 117, 220501 (2016).

41. Piltz, Ch., Sriarunothai, T., Ivanov, S. S., Wölk, S. \& Wunderlich, C. Versatile microwave-driven trapped ion spin system for quantum information processing. Sci. Adv. http://advances.sciencemag.org/content/2/7/e1600093 (2016).

42. Forn-Daz, P. et al. Observation of the Bloch-Siegert shift in a qubit-oscillator system in the ultrastrong coupling regime. Phys. Rev. Lett. 105, 237001 (2010).

43. Beaudoin, F., Gambetta, J. M. \& Blais, A. Dissipation and ultrastrong coupling in circuit QED. Phys. Rev. A 84, 043832 (2011).

44. Rossatto, D. Z., Villas-Bôas, C. J., Sanz, M. \& Solano, E. Spectral classification of coupling regimes in the quantum Rabi model. Phys. Rev. A 96, 013849 (2017).

45. Feranchuk, I. D., Komarov, L. I. \& Ulyanenkov, A. P. Two-level system in a onemode quantum field: numerical solution on the basis of the operator method. $J$. Phys. A 29, 4035 (1996).

46. Irish, E. K. Generalized rotating-wave approximation for arbitrarily large coupling. Phys. Rev. Lett. 99, 173601 (2007).

47. Gan, C. J. \& Zheng, H. Dynamics of a two-level system coupled to a quantum oscillator: transformed rotating-wave approximation. Eur. Phys. J. D. 59, 473-478 (2010).

48. Moya-Cessa, H., Jonathan, D. \& Knight, P. L. A family of exact eigenstates for a single trapped ion interacting with a laser field. J. Mod. Opt. 50, 265-273 (2003).

49. Moya-Cessa, H. Fast Quantum Rabi model with trapped ions. Sci. Rep. 6, 38961 (2016).

50. Peng, J., Ren, Z., Guo, G., Ju, G. \& Guo, X. Exact solutions of the generalized twophoton and two-qubit Rabi models. Eur. Phys. J. D. 67, 162 (2013).

51. Lo, C. F., Liu, K. L. \& Ng, K. M. The multiquantum Jaynes-Cummings model with the counter-rotating terms. Europhys. Lett. 42, 1 (1998). 
52. Olmschenk, S. et al. Manipulation and detection of a trapped $\mathrm{Yb}^{+}$hyperfine qubit. Phys. Rev. A 76, 052314 (2007).

53. Arrazola, l. et al. Pulsed dynamical decoupling for fast and robust two-qubit gates on trapped ions. Phys. Rev. A 97, 052312 (2018).

54. Zhong, H., Xie, Q., Batchelor, M. T. \& Lee, C. Analytical eigenstates for the quantum Rabi model. J. Phys. A 46, 415302 (2013).

55. Batchelor, M. T. \& Zhou, H.-Q. Integrability versus exact solvability in the quantum Rabi and Dicke models. Phys. Rev. A 91, 053808 (2015).

Open Access This article is licensed under a Creative Commons Attribution 4.0 International License, which permits use, sharing, adaptation, distribution and reproduction in any medium or format, as long as you give appropriate credit to the original author(s) and the source, provide a link to the Creative Commons license, and indicate if changes were made. The images or other third party material in this article are included in the article's Creative Commons license, unless indicated otherwise in a credit line to the material. If material is not included in the article's Creative Commons license and your intended use is not permitted by statutory regulation or exceeds the permitted use, you will need to obtain permission directly from the copyright holder. To view a copy of this license, visit http://creativecommons. org/licenses/by/4.0/.

c) The Author(s) 2018 